International Journal of Advanced Trends in Computer Science and Engineering

Available Online at http://www.warse.org/IJATCSE/static/pdf/file/ijatcse20832019.pdf

https://doi.org/10.30534/ijatcse/2019/20832019

\title{
Texture Filtration Module Under Stabilization Via Random Forest Optimization Methodology
}

\author{
${ }^{1}$ K Srinivasa Reddy, ${ }^{2}$ B. Suneela, ${ }^{3}$ Syed Inthiyaz, ${ }^{4}$ Sk. Hasane Ahammad, ${ }^{5}$ Gurram Narendra Santosh \\ Kumar, ${ }^{6} \mathbf{A}$ Mallikarjuna Reddy \\ ${ }^{1}$ Professor, ${ }^{1}$ Department of IT, Institute of Aeronautical Engineering, Hyderabad, India. \\ ${ }^{2,4,5}$ Research Scholar, ${ }^{2,3,4,5}$ Department of ECE, Koneru Lakshmaiah Engineering Foundation, Guntur, A.P, \\ India \\ ${ }^{3,6}$ Associate Professor, ${ }^{6}$ Department of CSE, Anurag Group of Institutions, Hyderabad.
}

\begin{abstract}
The three shading force esteems red/green as well as blue be picked up in a lone pixel on with a multisensor camera. The solitary feeler photography utilizes the shading channel group to getting simply a solitary power motivation in a pixel region. The changing of the indicated unrefined picture hooked on an occupied shading picture is described demosaicking. This document proposes a structure for amazing demosaicking united through the weight more than the data picture by means of a Random Forest Optimization (R-F-O) figuring. The demosaicking technique is gainful in be relevant the RFO wavelet strain to in a perfect sworld pick the coefficients as well as Customized Huffman Coding framework to the image previous to demo saicking. The projected framework is appraising through the Peak/Signal/Noise/Ratio, Structural Comparison Index Calculate also characteristic Comparison Index Determine regards furthermore have exhibited together sincerely moreover fairly to contain improved demosaicking grades. Finally existed estimations SA Adaptive/SA Universal/AF Demosaic/DDFAPD/MN Demosaic, along with AP Demosaic are differentiated and to count.
\end{abstract}

Key words: Random Forest Optimization (RFO), demosaicking, SA Universal, DDFAPD.

\section{INTRODUCTION}

Cameras are mainly ordinarily worn through every one of the general populations in their step by step time. The cams to facilitate are worn by the majority be the solitary sensor camera. This camera utilizes the shading channel display (C/F/A) to get the photos commencing nature. The channel permits simply a power a motivating force to subsist gotten in a lone pixel group. The picture got in such photography is recognized as the unrefined picture, which is also changed keen on an occupied shading picture beside the pipe-lined method described demosaicking. The additional a significant part of the time-worn $\mathrm{C} / \mathrm{F} / \mathrm{A}$ is the Bayer $\mathrm{C} / \mathrm{F} / \mathrm{A}$ including the green as well as the red-pixel regards in the odd lines along with blue also green pixel regards in the smooth segments. The Bayer configuration is delivered through Bayer [1] of the Eastman/ Kodak/ Company. Demosaicking counts container is heuristic in addition to non-heuristic in the environment. The heuristic strategy utilizes logical figuring associated with demosaicking, as well as on/heuristic philosophies, oversee crossbreed tally of demosaicking estimations.

Generally, demosaicking computations drop beneath the $2^{\text {nd }}$ arrangement (Fig/1). There are a couple of weight frameworks recommended on the C/F/A medley picture and demosaicked pictures. Pressing the photos are planned to rot the photos in command to lessen the abundance pixel regards in a picture. Weight includes II sorts: lossy as well as lossless; the past loses a number of pixels in sequence in the midst of the weight technique along with in lossless weight the representation is indistinct to the principal picture.

The coding abundance, interpixel reiteration, quantifiable overabundance as well as psycho physical unemployment are abstained from beside the weight strategies to decrease the number of bits in a representation. The move forwards in the state of-workmanship developments include provoked the rising of neural framework moreover feathery organizations in each part of investigating. There are a couple streamlining counts for convincing getting ready and conspicuous confirmation of the fittest regard or the perfect course of action. The nature-animated streamlining estimations are the progressing progress in headway in neural frameworks, for instance, Fruit Fly Optimization [2], Particle swarm improvement [3], Gray Wolf Optimization figuring [4] and much greater upgrade counts. The proposed work traces a selfassertive forest improvement (RFO) count which is capable of perceiving the coefficients and finds the fittest limit. This progression estimation is started into the weight strategies sought after by 
demosaicking to make an OK execution more than demosaicking. The story move towards includes the incredible generation of a filled shading picture in blend among the biorthogonal wavelet weight and improves the utilize of RFO count also Huffman Coding to gain improved weight coefficients so the changing is updated in addition to the execution of the demosaicking methodology is pushed ahead. The straggling leftovers of the manuscript are sorted out as seeks after. The second territory diagrams the associated employment sought after through the planned occupation in the the third fragment, $\mathrm{IV}^{\text {th }}$ segment shows the test outcomes, as well as examination moreover 5th portion, wraps up the document.

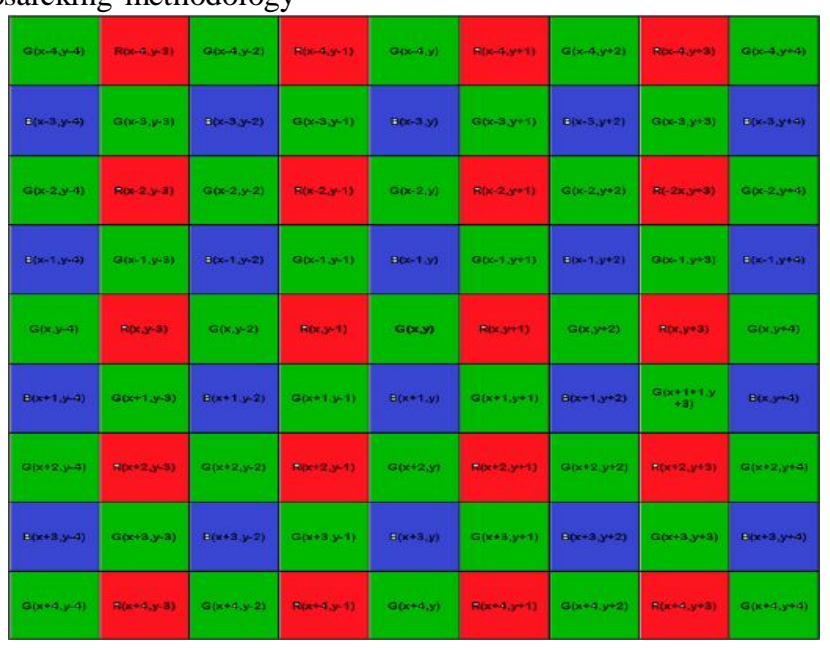

Figure 1: RGB Representation

\section{RELATED WORK}

There were a couple of demosaicking computations to push the projected employment [5] have completed three demosaicking systems subject to Taylor course of action; they are evaluatedorganize extrapolation, addition moreover cubic spine presentation nearby an edge-slanting classifier/ weighted centre channel to keep away from clouding at the edges [6] have imparted an edge liberated demosaicking figuring to facilitate joins judgment in the ways in addition to sheds taking tough choices [7] have recommended a multiscale shading slant demosaicking procedure to evaluate the heading of points reasonably moreover is gathered on the Bayer along with Lukac CFA on behalf of demosaicking [8] have shown an enhanced biased directional contribution through manhandling the solid shocking association unfriendly to partner constrained drive response channel and tendency weighted turn around channel in favor of the improvement of the demosaicking consequence [9] have abused the ghost relationship and interchannel connection be tween's the pixels of the picture also their nonclose-by personality closeness while the area geometry is dubious. A non-close-by isolating is worn to diminish the ancient pieces in the figure [10] have proposed a throwing a tally framework to pick the inclusion heading beside the edges in addition to a biased expansion in favor of demosaicking reliant on the interchannel tendency association inside the pixel of the image [11] have coupled the non-neighborhood method and meager depiction. A theatrical presentation is worn for denoising as the basic development as well as the comparable pieces are gathered Bynonneighborhood near patches sought after through a quadratic discipline work on behalf of upgrade [12] belongs to the essential in sequence moreover between channel relationship of the image; it is an iterative system of detaching the minimization convenience issue to settle combined definition [13], [14], [15] and [16] have superseded the shading refinement demosaicking through the waiting demosaicking in which the complexity flanked by the viewed and theoretical are assessed in the remaining region. The Laplacian regard is diminished unquestionably to smoothen the picture as well as the decline in the Laplacian regard nearby a directed channel provides enhanced demosaicking grades [17] utilizes the sub-band mix system on behalf of wavelets through abusing the elevated association connecting's wavelet sub/gatherings of dissimilar shading power regards [18] in attendance an perimeter adaptable computation that uses picture relationship along with Support/Vector/Machine by assigning the picture into flat furthermore border territories moreover taking care of them freely [19] include worn a multi-directional interposition which embeds in 8 different ways as well as is sophisticated through a prejudiced in reverse edge sifting[20] have executed a polynomial presentation in which the missteps are foreseen close by perimeter portrayal along with a biased entire procedure to decrease the relics [21] misuse a comprehensive demosaicking estimation relied 
upon between channel chrominance get [22] perceive the boundaries among an edge discoverer to decrease the darkening at the limits [23] have dispersed repeat of the heaps, and four includes four different ways are added to demosaic the image with a perfect cut off regard [24] contain measured together spatial proximity in addition to comparability amongst the pixels of the representation. Denoising of the demosaicked picture relies upon the complexity neural

\section{PROPOSED WORK}

The projected structure completes the $\mathrm{C} / \mathrm{F} / \mathrm{A}$ demosaicking among a fruitful wavelet weight close by the front line RFO [4] as well as the Huffman coding procedure. Fig-2 outlines the structure of the projected occupation. There are strategies with the aim of incorporate weight on the demosaicked picture or else more the medley pictures in favor of capability. The weighting methodology is worn earlier than demosaicking, which conveys enhanced demosaicking grades whilst differentiated and the standard figuring's. The picture is in a perfect world stuffed in any case by means of the wavelet weight along with Huffman coding. The decompressed picture is demosaicked via an evaluated-organized demosaicking count. The methods drew in with the anticipated employment be point by point underneath. The picture is rotted keen on Towering Pass along with the Low Pass channels. The limits contain elevated repeat moreover flat areas include short repeat coefficients. The significance of the coefficients facilitates in perceiving the boundaries in the wavelet area [27] (Figure 3). The projected occupation has a lossless weight method among framework, which is the standout amongst the newest examples in picture changing [25] use a significant outstanding estimation and multi-show mix to reduce shading old rarities started in the wake of demosaicking commonly with an adaptable demosaicking figuring [26] have stuffed the mosaic pictures through the Golomb-/Rice programming procedure towards empty redundancies in the spatial in addition to ghost region.

biorthogonal wavelet change. A lone extent limit is superseded beside two scaling limits in biorthogonal wavelet change. The essentialness is centered around single two or three the coefficients. Biorthogonal wavelet gives an additional dimension of a chance than previous orthonormal changes. The supposition regards are in a base choice in addition to the nuances of the representation are secured in command to hold the nuances of the representation. Fig/4 portrays the vertical, corner to corner moreover level coefficient nuances of the image on concerning the biorthogonal wavelet change for picture weight. The colossal proportion of parameters are completed nothing in support of the strain to subsist powerfully. An indispensable proportion of null helpers in the lossless weight; concerning $43 \%$ of the coefficients be changed over to zero in support of weight. The coefficients are upgraded by RFO.

\section{RFO:}

This belongs to acme predators of the sequence

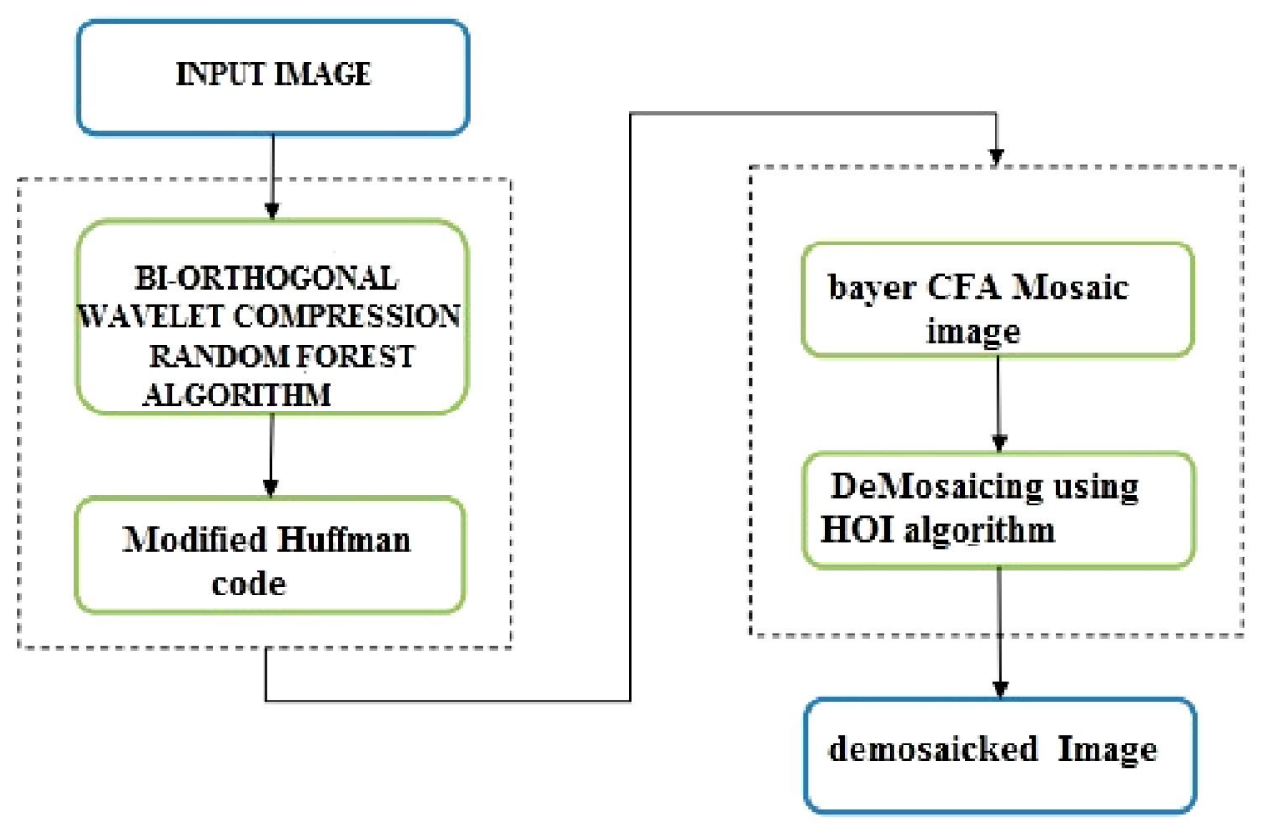

Figure 2: Random forests based proposed work 
Subjective Forest is an adaptable, simple to utilize AI computation with the aim of creates, still devoid of hyper-parameter alteration, a phenomenal outcome as a law. It is furthermore a supporter surrounded by the generally worn counts, given that it's straightforwardness and the manner in which that it will, in general, be used for both gathering and backslide endeavors. Sporadic Forest is a coordinated learning figuring. Like you would as of now have the capacity to see from its name, it makes timberland and makes it somehow subjective. The ,forest" it collects, is a gathering of Decision Trees, as a general rule arranged with the "sacking" technique. The general idea of the pressing system is that a mix of learning models grows the general result. Unpredictable timberland produces various decision trees and merges them to get a dynamically accurate and stable desire. One noteworthy good position of the sporadic forest is, that it might be used for both requests and backslide issues, which shape the prevailing piece of current AI structures. I will talk about discretionary timberland in course of action since portrayal is a portion of the time considered the structure square of AI. Underneath you can see how sporadic timberland would look like with two
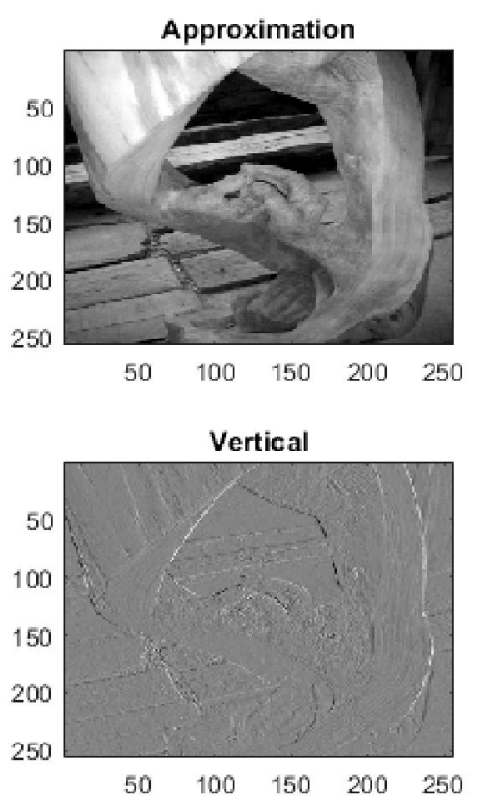

trees: Random Forest has nearly the comparable hyper parameters as a decision tree or a stowing classifier. Fortunately, you don't have to combine a decision tree with a sacking classifier and can just easily use the classifier-class of Random Forest. Like I recently expressed, with Random Forest, you can in like manner oversee Regression assignments by using the Random Forest regressor. Unpredictable Forest adds additional intervention to the model while building up the trees. Instead of filtering for the most fundamental component while section a center point, it searches for the best part among a self-assertive subset of features. This results in a wide different assortment that generally results in an unrivaled model. In this way, in Random Forest, simply a subjective subset of the features is thought about by the figuring on behalf of section a center point. You can still create trees progressively self-assertive, by moreover via sporadic edges in favor of every segment as opposed to chasing down the best edges.

$$
[\mathrm{cA}, \mathrm{cH}, \mathrm{cV}, \mathrm{CD}]=\mathrm{dwt} 2(\mathrm{X}, \text { 'sym4','mode','per') }
$$
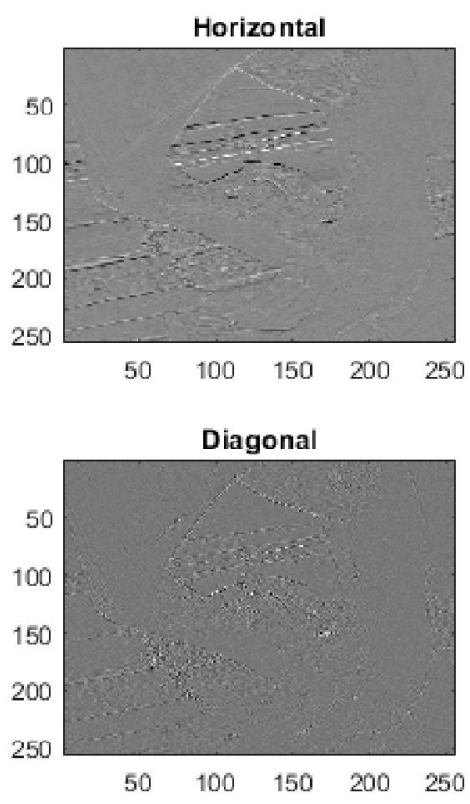

Figure 3: Bi-orthogonal wavelet convert of level 1 as well as level 2 horizontal, diagonal moreover vertical detailed subimages. 


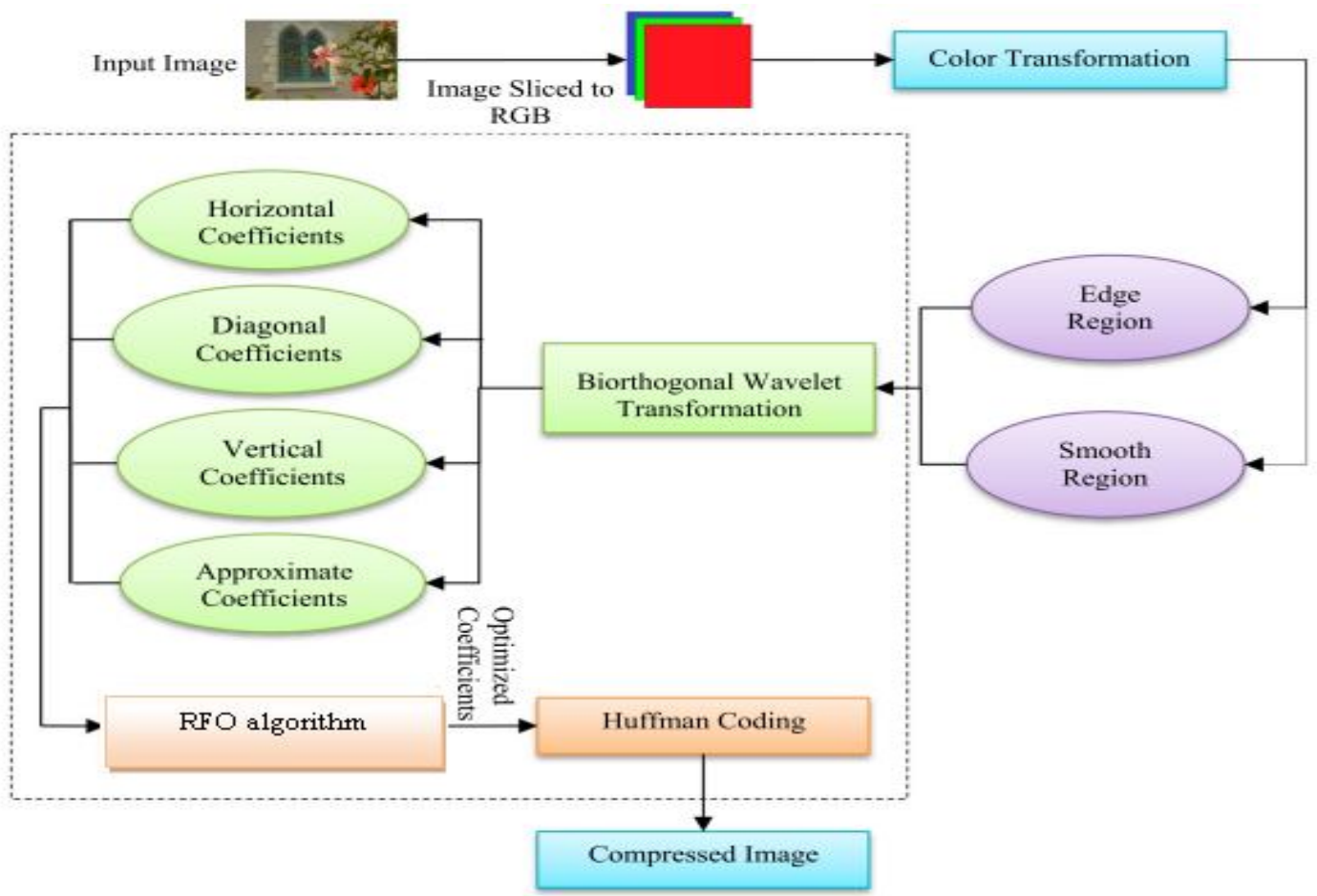

Figure 4: Optimized structure using RFO AND Huffman coding

Arbitrary woods (RF) build numerous individual choice trees at preparing. Expectations commencing all trees are pooled to create the last forecast; the method of the classes on behalf of order or the mean expectation for relapse. As they utilize an accumulation of results to settle on an ultimate conclusion, they be alluded to as Ensemble strategies.

$$
n i_{j}=w_{j} C_{j}-w_{\text {left }(j)} C_{l e f t(j)}-w_{\text {right }(j)} C_{\text {right }(j)}
$$

Highlight significance is determined as the decline in hub contamination prejudiced through the likelihood of achieving that hub. The hub likelihood container is determined through the number of tests that achieve the hub, partitioned through the aggregate digit of tests. The advanced the esteem the additional imperative the element.

$>$ ni $\operatorname{sub}(\mathrm{j})=$ the significance of hub $\mathrm{j}$

$>\quad \mathrm{w} \operatorname{sub}(\mathrm{j})=$ weighted figure of tests achieving hub $\mathrm{j}$

$>\mathrm{C} \operatorname{sub}(\mathrm{j})=$ the pollution estimation of hub $\mathrm{j}$

$>\operatorname{left}(\mathrm{j})=$ kid hub starting left split on hub $\mathrm{j}$

$>\operatorname{right}(\mathrm{j})=$ kid hub commencing right split on hub $\mathrm{j}$

The significance for each element on a choice tree is then determined as:

$$
\begin{gathered}
f i_{i}=\frac{\sum_{j: \text { node } j \text { splits on feature } i} n i_{j}}{\sum_{k \in \text { all nodes }} n i_{k}} \\
>\quad \text { fi sub(i)= the significance of feature } \mathrm{i} \\
>\quad \text { ni sub(j)= the importance of node } \mathrm{j} \\
\text { norm } f i_{i}=\frac{f i_{i}}{\sum_{j \in a l l ~ f e a t u r e s} f i_{j}}
\end{gathered}
$$

These would then be able to be standardized to an incentive somewhere in the range of 0 as well as 1 by isolating by the entirety of all element significance esteems: The last component significance, at the Random Forest dimension, is it's normally more than every one of the trees. The total of the component's significance esteem on every tree is determined moreover partitioned through the aggregate figure of trees:

$$
\text { RFfi }_{i}=\frac{\sum_{j \in \text { all trees }} \text { norm } f i_{i j}}{T}
$$

$>$ RFfi $\operatorname{sub}(\mathrm{i})=$ the significance of characteristic I considered commencing all trees in the Random Forest model

$>$ normal $\operatorname{sub}(\mathrm{IJ})=$ the normalized characteristic consequence on behalf of I in tree $\mathrm{j}$

$>\mathrm{T}=$ total figure of trees 

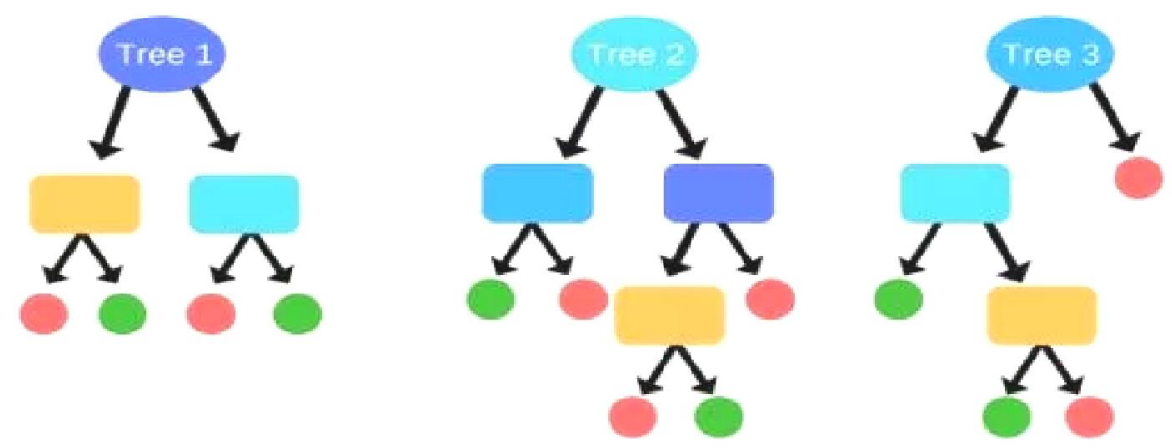

Figure 5: Decision of tree diagram

Irregular timberland is just a gathering of choice trees whose outcomes are amassed into one last outcome. Their capacity to confine over fitting without generously expanding mistake because of predisposition is the reason they are such amazing models. One-way Random Forests diminish change is via preparing on various examples of the information.

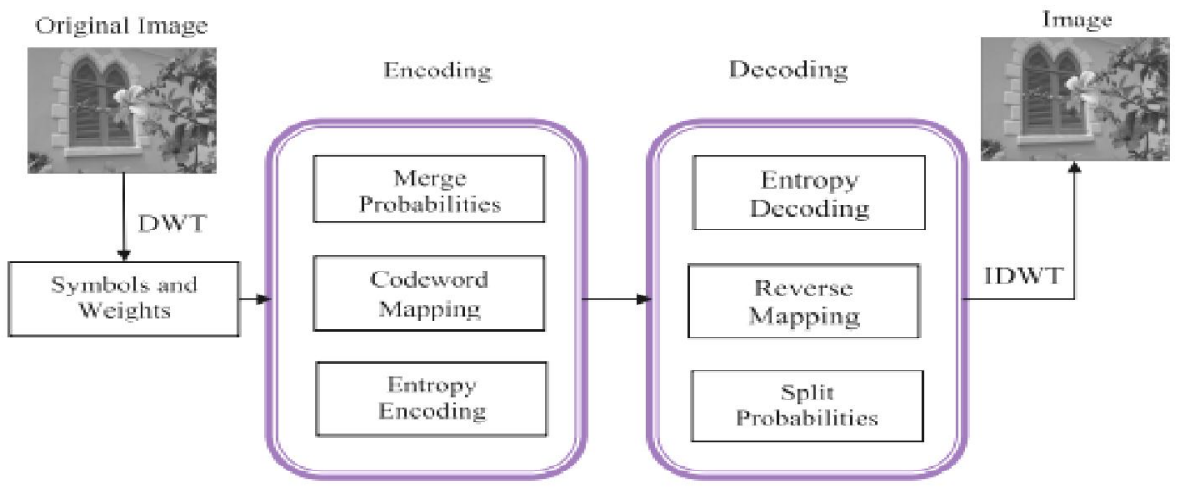

Figure 6: Encoding and decoding process

The images also their loads (i.e. pixel esteems as well as the event) be organized in the successive request base up. The pixel through the most noteworthy event has the littlest code phrase, as well as the pixels among the minimum event, contain the biggest code word. The excess is diminished beside blending the probabilities of the pixels contains the most reduced event iteratively pending the point when just 2 probabilities are left along with the objective is accomplished. The code words are mapped to the pixel esteems towards getting the packed picture in the wake of applying the biorthogonal wavelet change. An entropy encoder is utilized to get the ideal code in favor of the pixel along with their events as in Eqn(12) (Figure-6).

$$
\text { Entropy }=-\sum_{s} P(s) \log _{2}(s)
$$

\section{HIGH-ARRANGE DEMOSAICKING}

Demosaicking is the way toward evaluating the gone astray power estimation of the crude picture towards a total otherwise a filled shading picture.
The packed picture is demosaicked beside a 4 directional demosaicking over the limits as well as not beside the edges [5]. The picture is remade by using the packed picture got after the above procedure. The picture is changed over to a mosaic so as to demosaic the packed picture to acquire a superior remade picture with viable outcomes. The demosaicking procedure is partitioned into green force esteem demosaicking along with red/blue power esteem demo/saicking [33].

\section{GREEN POWER ESTEEM DEMOSAICKING}

The most ideal inexact of the not their force esteem is dictated through the closest realized pixel esteems. The demosaicking is completed alongside the edges along with over the inside pixel arrange Ix,y so as to acquire progressively exactapproximate in favor of demosaicking. The high order subsidiary is evaluated through utilizing the Taylor arrangement [30] with the end goal to facilitate the misplaced green diverts is recognized in the focused blue area. 


$$
g(x, y)=\sum_{m n=0}^{\infty} \frac{g^{m n}(i, j)}{m n !}(x, y)-(i, j)^{m n}
$$

An important dissimilarity opinion is exploited, where

$$
g^{\prime}(x, y)=\frac{g(x, y+1)-g(x, y-1)}{2}
$$

The $2^{\text {nd }}$ charge derivative is specified by Equation-as:

$$
\begin{aligned}
\hat{G}_{x, y}= & G_{x, y-1}+g^{\prime}(x, y-1)+\frac{1}{8}(g(x, y+1) \\
& -2 g(x, y-1)+g(x, y-3)) \\
\hat{G}_{x, y}= & G_{x, y-1}+\frac{1}{2}\left(B_{x, y}-B_{x, y-2}\right)+\frac{1}{8}\left(\hat{G}_{x, y+1}\right. \\
& \left.-2 \hat{G}_{x, y-1}+\hat{G}_{x, y-3}\right)
\end{aligned}
$$

Where,

$$
g(x, y-1)=\frac{B_{x, y}-B_{x, y-2}}{2}
$$

The pixel to facilitate is more distant beginning the objective pixel is wiped out. Loads of the most distant pixel be decreased as well as increment the loads of the closest pixel esteem. It delivers a superior gauge beside the edge. The green force esteem demosaicking is scientifically communicated through Equations:

$$
\begin{aligned}
& \hat{G}_{x, y}^{N}=G_{x-1, y}+\frac{1}{2}\left(B_{x, y}-B_{x-2, y}\right)+\frac{1}{8}\left(G_{x+1, y}\right. \\
& \left.-2 G_{x,-1 y}+G_{x-3 y}\right) \\
& \hat{G} r_{x, y}^{s}=G r_{x+1, y}+\frac{1}{2}\left(B l_{x y}-B l_{x+z y}\right)+\frac{1}{8}\left(G r_{x-1, y}\right. \\
& \left.-2 G r_{x+1, y}+G r_{x+3 y}\right) \\
& G_{x, y}^{E}=G_{x y+1}+\frac{1}{2}\left(B_{x y}-B_{x y+2}\right)+\frac{1}{8}\left(G_{x y-1}\right. \\
& \left.-2 G_{x, y+1}+G_{x y+3}\right) \\
& \hat{G}_{x, y}^{W}=G_{x, y-1}+\frac{1}{2}\left(B_{x, y}-B_{x y-2}\right)+\frac{1}{8}\left(G_{x, y+1}\right. \\
& \left.-2 G_{x, y-1}+G_{x, y-3}\right)
\end{aligned}
$$

The exceeding equations are imitatively commencing the important estimate of the green strength price in 4 instructions $(\mathrm{N}, \mathrm{S}, \mathrm{E}$, and $\mathrm{W})$.

\section{RED/BLUE INTENSITY VALUE DEMOSAICKING}

The main request gauge is utilized on behalf of the red along with blue power esteem demosaicking, while the high-arrange gauge is used for the green force esteem demosaicking. The red along with the blue power esteem is beneath examined to an expansive degree plus the exactness can't be enhanced additional through high-arrange demosaicking. The demosaicking is evaluated in 8 ways on behalf of the red/blue demosaicking. The red force an incentive in the blue as well as green areas as well as blue power an incentive in the red also green areas are recognized through EqNs (22)(29).

$$
\begin{aligned}
& \hat{R} d_{x, y-1}^{N}=\hat{R} d_{x-1, y-1}+\left(G r_{x, y-1}-\hat{G} r_{x-1, y-1}\right) \\
& \hat{R} d_{x, y-1}^{s}=R d_{x+1, y-1}+\left(G r_{x, y-1}-G r_{x+1, y-1}\right)
\end{aligned}
$$

The beyond conditions are rehashed in favor of the blue (Bl) force an incentive in the green moreover red areas of the picture. On inserting the three force esteems independently subsequent to pressure, a totally demosaicked pictures in support of the three power esteem are acquired(34).

$$
\begin{gathered}
\hat{R} d_{x y}^{N W}=\hat{R} d_{x-1, y-1}+\left(\hat{G} r_{x, y}-\hat{G} r_{x-1, y-1}\right) \\
\hat{R} d_{x, y}^{N E}=R d_{x-1, y+1}+\left(\hat{G} r_{x, y}-\hat{G} r_{x-1, y+1}\right) \\
\hat{R} d_{x, y}^{S W}=R d_{x+1, y-1}+\left(\hat{G} r_{x, y}-\hat{G} r_{x+1, y-1}\right) \\
\hat{R} d_{x, y}^{S E}=R d_{x+1, y+1}+\left(\hat{G} r_{x, y}-\hat{G} r_{x+1, y+1}\right) \\
\hat{R} d_{x, y-1}^{E}=R d_{x, y}+\left(G r_{x, y-1}-G r_{x y y}\right) \\
\hat{R} d_{x, y-1}^{W}=R d_{x y-2}+\left(G r_{x y-1}-\hat{G} r_{x y-2}\right)
\end{gathered}
$$




\section{RESULTS}

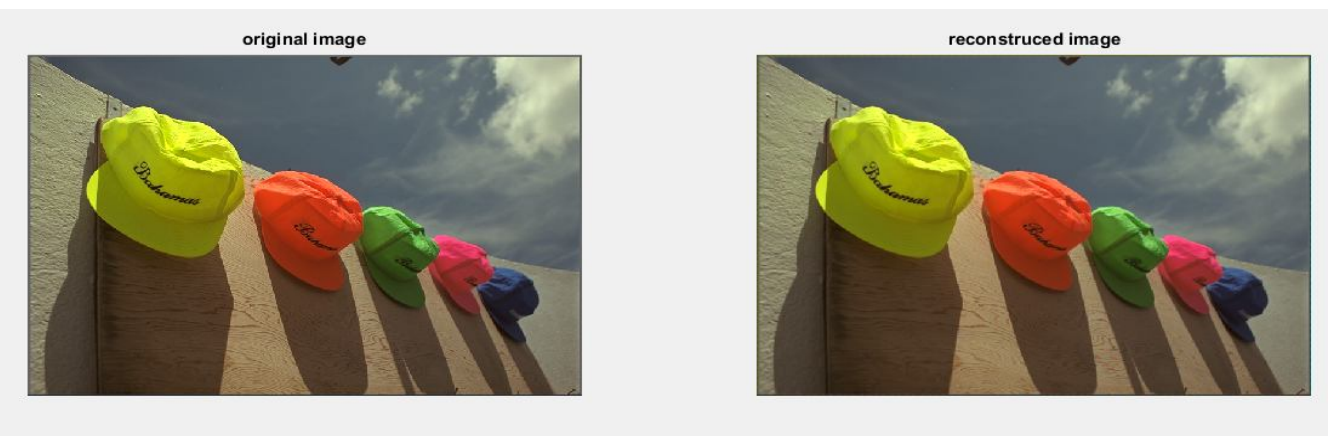

Figure 7: Original vs reconstruction images

Fig.7 shows that demosaicking of proposed method output here $1^{\text {st }}$ one is the original image and $2^{\text {nd }}$ is $R F O$ output image using proposed trees we achieve the good improvement

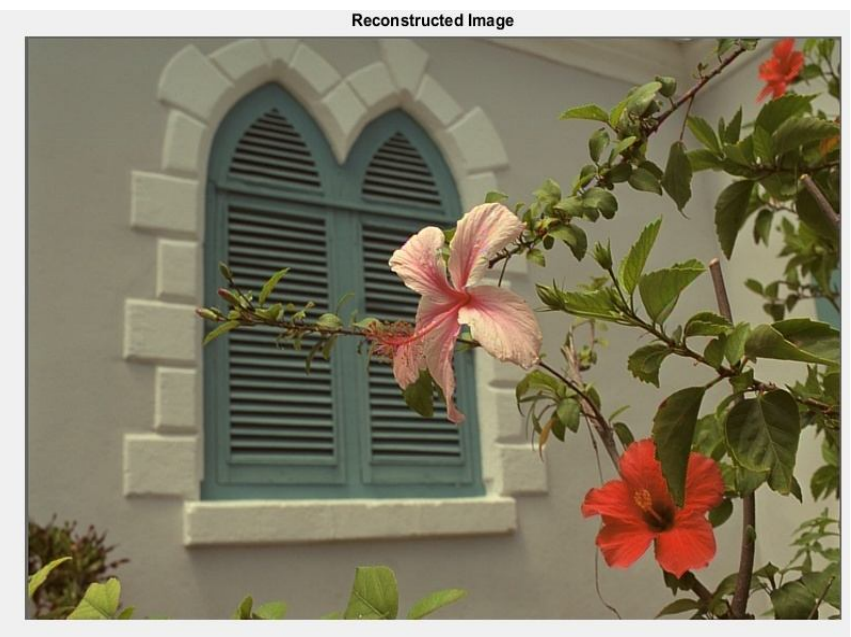

Figure: 8 Demosaicking image

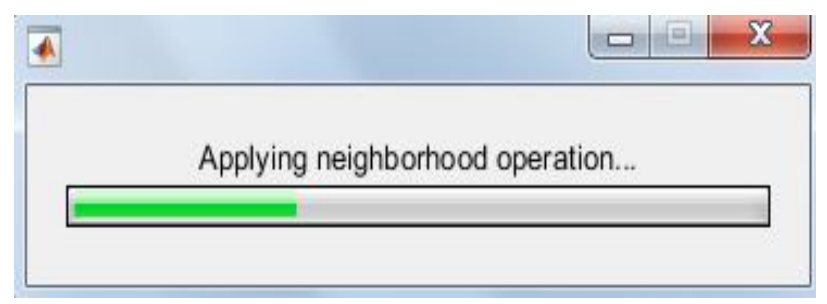

Figure: 9 Processing using RFO

Fig.8 \& 9 explains that demosaicking processing and output of RFO based on proposed tree architecture here we got best clarity image by applying true tree on each iteration

Clmsap clmax $=2 \operatorname{mmax}=2$ nmax $=2$ depth $\max$ $=2$

Subscripted assignment dimension mismatch.
Error in clmap (line 53) PQ (m, :)=parent.PQ;

>> branch

Error using branch (line 15)

Not enough input arguments.

>> clmap clmax $=5 \operatorname{mmax}=10$ nmax $=100$ depthmax $=20$ 


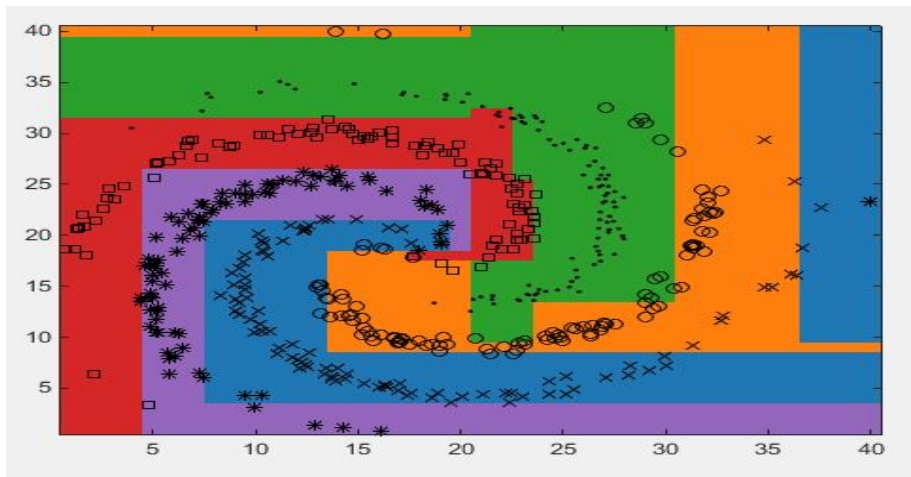

Figure 10: Random forest pixels pointing

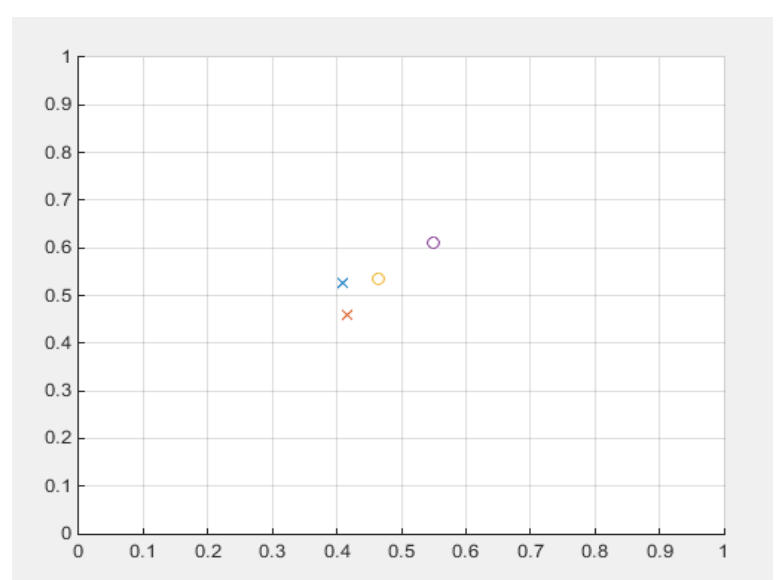

Figure: 11 Pixels fixing in the MATLAB window

Fig.10.11 shows that pointing random image on proposed iteration here $2^{\text {nd }}$ figure explains that tree true or false classification based on a random search.

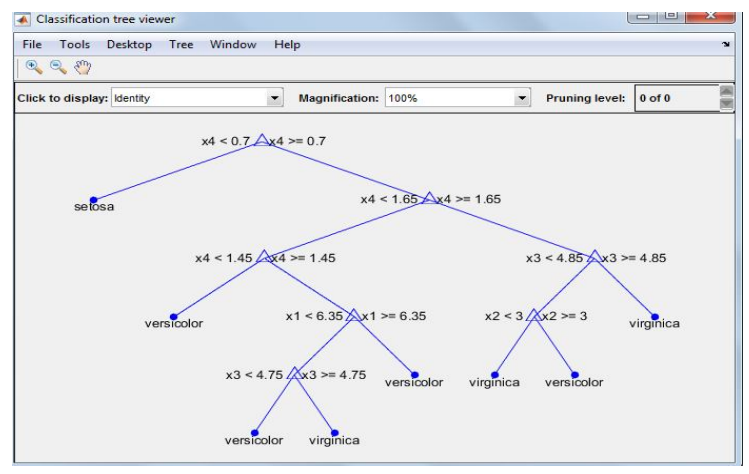

Figure: 12 Tree representation

Figure 12.explains that depending upon tree value gave the false or true justification on data base images

\section{Tree Bagger}

Ensemble with 60 bagged decision trees:
Training X:

[150x4]

Training Y:

[150x1]

Method: classification

Nvars:

4 


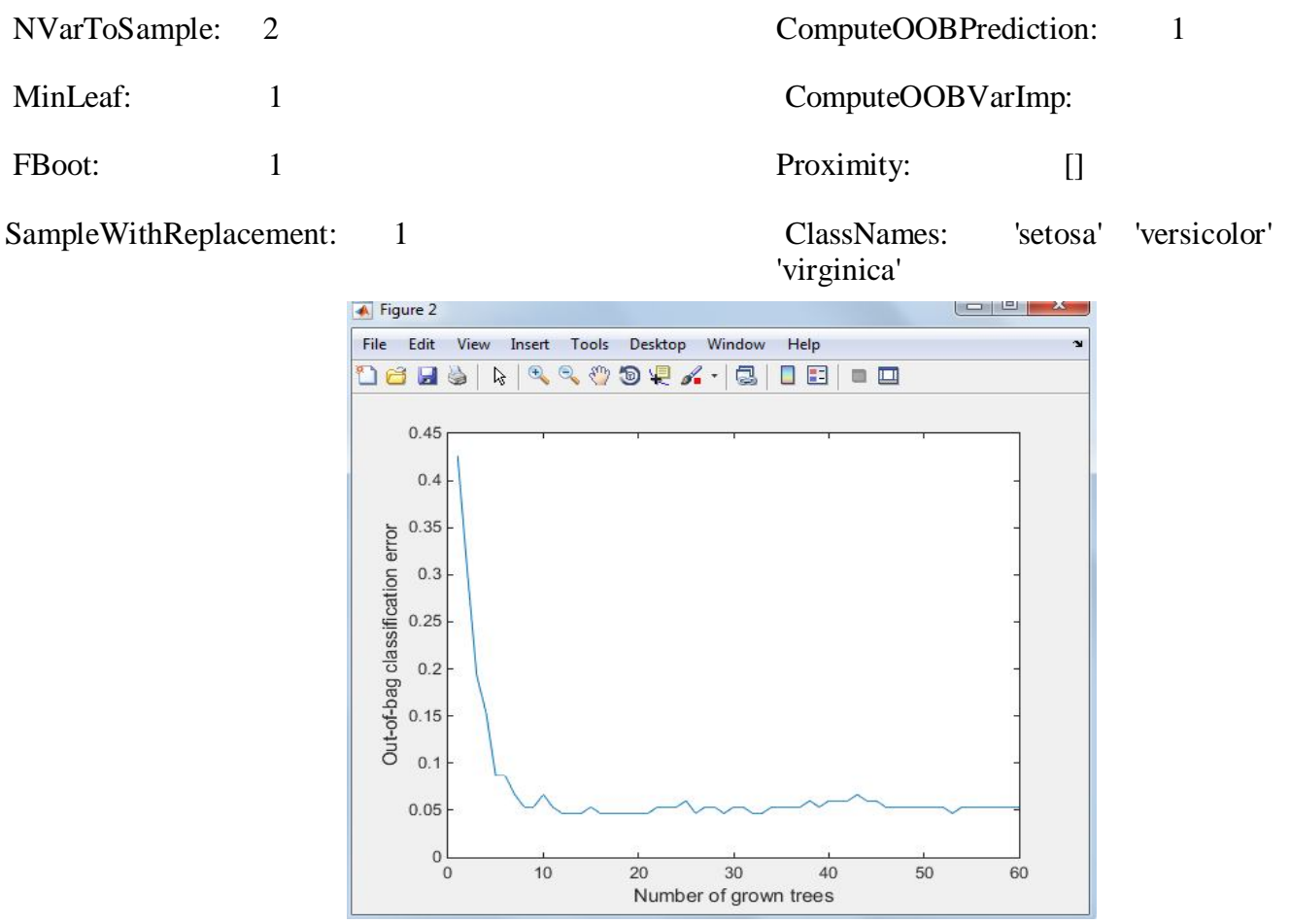

Figure 13: trees classification

Table 1: Comparison table for MSE

\begin{tabular}{|c|c|c|c|c|c|c|c|c|c|}
\hline \multirow[t]{3}{*}{ Image } & \multirow{2}{*}{\multicolumn{3}{|c|}{$\frac{\text { SA Adaptive }}{\text { MSE }}$}} & \multirow{2}{*}{\multicolumn{3}{|c|}{$\begin{array}{c}\text { SA Universal } \\
\text { MSE }\end{array}$}} & \multirow{2}{*}{\multicolumn{3}{|c|}{$\begin{array}{c}\text { Random forest } \\
\text { MSE }\end{array}$}} \\
\hline & & & & & & & & & \\
\hline & Red & Green & Blue & Red & Green & Blue & Red & Green & Blue \\
\hline 1 & 1.814230 & 0.873230 & 1.49084 & 1.845960 & 0.922660 & 1.574750 & 1.365270 & 0.45410 & 1.050880 \\
\hline 2 & 2.611620 & 1.425380 & 2.97671 & 2.586730 & 1.532210 & 2.850230 & 2.682430 & 1.744910 & 2.689950 \\
\hline 3 & 1.887790 & 1.148690 & 1.28136 & 1.965160 & 1.236630 & 1.331290 & 1.353420 & 0.513210 & 1.323360 \\
\hline 4 & 5.295770 & 2.193280 & 4.23596 & 5.450820 & 2.699430 & 4.281540 & 5.296330 & 2.379140 & 4.903670 \\
\hline 5 & 1.69790 & 0.748480 & 1.43678 & 1.777960 & 0.858290 & 1.52290 & 1.24570 & 0.532830 & 1.165810 \\
\hline 6 & 2.704250 & 1.42840 & 1.79725 & 2.786350 & 1.548740 & 1.722960 & 2.285740 & 1.326230 & 1.542550 \\
\hline 7 & 2.82390 & 2.045280 & 2.52528 & 1.814010 & 0.78270 & 1.422830 & 1.159830 & 0.870170 & 1.489370 \\
\hline 8 & 1.797140 & 0.774790 & 1.45124 & 1.843120 & 1.19630 & 1.855380 & 1.544570 & 1.174010 & 1.584470 \\
\hline 9 & 1.738370 & 1.063890 & 1.79439 & 1.600560 & 1.273730 & 1.63160 & 1.324880 & 0.812760 & 1.297610 \\
\hline 10 & 1.535090 & 1.206690 & 1.5954 & 2.492230 & 1.593660 & 2.611480 & 2.093520 & 1.680670 & 2.338680 \\
\hline
\end{tabular}

Table 2: Comparison table for PSNR

\begin{tabular}{|c|c|c|c|c|c|c|c|c|c|}
\hline \multirow{2}{*}{ Image } & \multicolumn{3}{|c|}{ 'SA Adaptive' } & \multicolumn{3}{c|}{ 'SA Universal' } & \multicolumn{3}{c|}{ 'Random forest' } \\
\cline { 2 - 10 } & \multicolumn{3}{|c|}{ PSNR } & \multicolumn{3}{c|}{ PSNR } & \multicolumn{3}{c|}{ PSNR } \\
\cline { 2 - 10 } & Red & Green & Blue & Red & Green & Blue & Red & Green & Blue \\
\hline 1 & 45.54426 & 48.7159 & 46.39694 & 45.468580 & 48.480410 & 46.158690 & 48.715470 & 52.694940 & 49.520510 \\
\hline 2 & 43.96145 & 46.5951 & 43.39354 & 44.003290 & 46.277620 & 43.582 & 45.686560 & 47.471060 & 45.674840 \\
\hline 3 & 45.37028 & 47.52867 & 47.05478 & 45.196830 & 47.208390 & 46.88808 & 48.506880 & 52.254710 & 48.597650 \\
\hline 4 & 40.89134 & 44.71968 & 41.86124 & 40.766190 & 43.818070 & 41.8148 & 42.809820 & 46.18780 & 43.137920 \\
\hline 5 & 45.82941 & 49.38889 & 46.5596 & 45.631590 & 48.794470 & 46.30409 & 48.841330 & 52.117970 & 44.107120 \\
\hline 6 & 43.8150 & 46.5832 & 45.58417 & 43.680440 & 46.231010 & 45.76805 & 46.354560 & 48.58890 & 47.975610 \\
\hline 7 & 43.62060 & 45.02372 & 44.10717 & 45.544410 & 49.194850 & 46.59929 & 48.127670 & 50.262330 & 49.027040 \\
\hline 8 & 45.58121 & 49.23887 & 46.5134 & 45.475260 & 47.352290 & 45.44648 & 48.970270 & 49.079070 & 47.866180 \\
\hline 9 & 45.72946 & 47.86158 & 45.59156 & 46.088090 & 47.080040 & 46.00465 & 47.249880 & 49.527220 & 48.676960 \\
\hline 10 & 46.26969 & 47.31468 & 46.10249 & 44.164930 & 46.106860 & 43.96194 & 46.719380 & 47.624970 & 46.259260 \\
\hline
\end{tabular}


Table 1, 2 explains that the proposed method got fewer signify square mistake also elevated peak to signal noise ratio based on this clearly said that random forest optimization is a good method in the analysis of demosaicking .

\section{CONCLUSION}

In this document, we recommend a shading interjection strategy on behalf of Bayer channel cluster pictures dependent on RFO. Testing a serial of LIVE standard pictures by contemplating multichannel relationship and also including increasingly sensible edge location bearings while evaluating the missing shading segments, the proposed addition technique has been demonstrated

\section{REFERENCES}

[1] Bayer BE. Color imaging array. The United States Patent 3,971,065. 1976.

[2] Xing B, Gao W-J. Fruit Fly optimization algorithm innovative computational intelligence. A rough guide to 134 clever algorithms. Intell Syst Ref Libr. 2014; 62:167170. DOI:10.1007/978-3-319 03404-1.

[3] Wang D, Tan D, Liu L. Particle swarm optimization algorithm: an overview. Soft Comput. 2017. DOI:10.1007/ s00500-0162474-6.

[4] Mirjalili S, Mohammad S, Lewis A. Grey wolf optimizer. Adv Eng Softw. 2014; 69:46-61. DOI: 10.1016/j.advengsoft. 2013.12.007.

[5] Li JS, Randhawa S. Color filter array demosaicking using high-order interpolation techniques with a weighted median filter for sharp color edge preservation. IEEE Trans Image Process. 2009; 18:1946-1957. DOI:10.1109/ TIP.2009.2022291.

[6] Pekkucuksen I, Altunbasak Y.Gradient-based threshold free color filter array interpolation, in the international conference on image processing.ICIP.2010, DOI:10.1109/ICIP. 2010.5654327.

[7] Pekkucuksen I, Altunbasak Y. Multiscale gradients-based color filter array interpolation. IEEE Trans Image Process. 2013; 22:157-165. DOI:10.1109/TIP.2012.2210726.

[8] He L, Chen X, Jeon G, et al. Improved directional weighted interpolation method for single-sensor camera imaging. Opt Eng. 2014;53:093103.DOI:10.1117/1.OE.53.9.09310 3.

[9] Duran J, Buades A. Self-Similarity and spectral correlation adaptive algorithm for color demosaicking. IEEE Trans Image Process. 2014; 23:4031-4040.

[10]Chen X, Jeon G, Jeong J. Voting-Based directional interpolation method and Its application to still color image demosaicking. that a perfect enhanced visualization of the remade picture with higher target PSNR esteem could be accomplished. In the interim, it makes a decent harmony between computational multifaceted nature and nature of picture after introduction. Be that as it may, it ought to likewise be noticed that the tried pictures are so perfect without thinking about impedance from internal clamors. In addition, the calculation will be progressively proficient if the heading tree is utilized during the time spent adding the missing red/blue channel. All in all, because of its low unpredictability and great insertion execution, the newly proposed technique is appropriate for Bayer CFA pictures demosaicking.

IEEE Trans Circuits Syst Video Technol. 2014; 24:255-262 DOI:10.1109/TCSVT.2013.225542 1.

[11] Shi J, Wang C, Zhang S. Region-adaptive demosaicking with weighted values of multidirectional information. $\mathrm{J}$ Commun. 2014; 9:930-936. DOI:10.12720/jcm.9.12.930936.

[12]Chang K, Ding PLK, Li B. Color image demosaicking using inter-channel correlation and nonlocal self-similarity. Signal Process Image Commun. 2015;39(Part A):264-279. DOI: $10.1016 /$ j.image.2015.10.003. Figure 10 . Comparative analysis of the average FSIM values. THE IMAGING SCIENCE JOURNAL 9.

[13] Kiku D, Monno Y, Tanaka M, et al. Minimized-Laplacian residual interpolation for color image demosaicking. Proceedings of SPIE-IS\&T Electronic Imaging; s2014. DOI: 10.1117/ 12.2038425.

[14] Ye W, Ma KK. Color image demosaicing using iterative residual interpolation. IEEE Trans Image Process. 2015; 24:5879-5891. DOI:10.1109/TIP.2015.2482899.

[15] Kim Y, Jeong J. Four-Direction residual interpolation for demosaicking. IEEE Trans Circuits Syst Video Technol. 2016; 26:881890. DOI: 10.1109/TCSVT.2015.2428552.

[16] Jeon G, Anisetti M, Wang L, et al. Locally estimated heterogeneity property and its fuzzy filter application for deinterlacing.Inf Sci.2016;354:112-130.DOI:10.1016/j. ins.2016. 03.016.

[17] Shao P, Ding S, Ma L, et al. Edge-preserving image decomposition via joint weighted least squares. Comput Vis Media. 2015; 1:37-47. DOI: 10.1007/s41095- 015-0006-4.

[18] Jia X, Zhao B, Zhou M, et al. An edgeadaptive demosaicking method based on image correlation. J Cent South Univ. 2015; 22:1397-1404. DOI:10.1007/s11771-015-26579. 
[19] Chen X, He L, Jeon G, et al. Multidirectional weighted interpolation and refinement method for Bayer pattern CFA demosaicking. IEEE Trans Circuits Syst Video Technol.2015; 25:1271-1282.DOI:10.1109

[20] Wu J, Anisetti M, Wu W, et al. Bayer Demosaicking with polynomial interpolation. IEEE Trans Image Process. 2016; 25:53695382. DOI:10.1109/TIP.2016.2604489.

[21]Zhang C, Li Y, Wang J, et al. "Universal demosaicking of color filter arrays". IEEE Trans Image Process. 2016; 25:5173-5186. DOI:10.1109/TIP.2016.2601266.

[22]Lien CY, Yang FJ, Chen PY. An efficient edge-based technique for color filter array demosaicking. IEEE Sens J. 2017; 17:40674074. DOI:10.1109/JSEN.2017.2706086.

[23] Li N, Li JSJ, Randhawa S. Color filter array demosaicking based on the distribution of directional color differences. IEEE Signal Process Lett. 2017; 24:604-608. DOI:10.1109/LSP.2017.2658667.

[24] Wang J, Wu J, Wu Z, et al. Bilateral filtering and directional differentiation for Bayer demosaicking. IEEE Sens J. 2017; 17:726-734. DOI:10.1109/JSEN.2016. 2623422.

[25]Tan DS, Chen W-Y, Hua K-L. Deep demosaicking: adaptive image demosaicking via multiple deep fully convolutional networks. IEEE Trans Image Process. 2018; 27:2408-2419.

DOI:10.1109/TIP.2018.2803341.

[26]Zhang N, Wu X. Lossless compression of color mosaic images. IEEE Trans Image Process. 2006; 15:1379-1388.

[27]Calderbank AR, Daubechies I, Sweldens W, et al. Wavelet transforms that Map integers to integers. Appl Comput Harmon Anal. 1998; 5:332-369.

[28] Tizhoosh HR. (2005). Opposition-based learning: a new scheme for machine intelligence, International Conference on Computational Intelligence for Modelling, Control and Automation, and International Conference on Intelligent Agents, Web Technologies and Internet Commerce.
[29]Huffman DA. (1952). A method for the construction of minimum-redundancy codes, Proceedings of the I.R.E. A. p. 1098-1101.

[30] Kreyszig E. (2006). Advanced engineering mathematics.

[31] Ibrahim Pekkucuksen YA. (2010). Gradientbased threshold free color filter array interpolation. Proceedings of the IEEE International Conference on Image Processing. p. 137-140.

[32] Kiku D, Monno Y, Tanaka M, et al. (2013). Residual Interpolation for Color Image Demosaicking. Proceedings of the International Conference on Image Processing. ICIP 2304 2308. DOI:10.1109/TIP.2016.2518082.

[33] Rama Chandra Manohar, K., S. Upendra, V. Durgesh, B. Sandeep, K. S. K. Mallik, G. N. S. Kumar, and S. H. Ahammad. 2018. "Modeling and Analysis of Kaplan Turbine Blade using CFD." International Journal of Engineering and Technology (UAE) 7 (3.12 Special Issue 12): 10861089. www.scopus.com. https://doi.org/10.14419/ijet.v7i3.12.17766

[34] Vijaykumar, G., A. Gantala, M. S. L. Gade, P.Anjaneyulu, and S. H. Ahammad. 2017."Microcontroller Base Heartbeat Monitoring and Display on PC." Journal of Advanced Research in Dynamical and ControlSystems9(4):250-260.

[35] Tirthani Ganesan, Neha R. "Data Security in Cloud Architecture Based on Diffie Hellman and Elliptical Curve Cryptography."

[36] As 'habi, Keivan, Arman Vafabakhsh, and Saeed Borji. 2016. "DATA TRANSMISSION SECURITY IN CLOUD COMPUTING." Indian Journal of Fundamental and Applied Life Sciences 6: 2231-6345 